\title{
Relaciones Públicas y
}

Sustentabilidad: estudio en

organizaciones brasileñas y

ecuatorianas

\section{Public relations and sustainability. A study of Brazilian and Ecuadorian organisations}
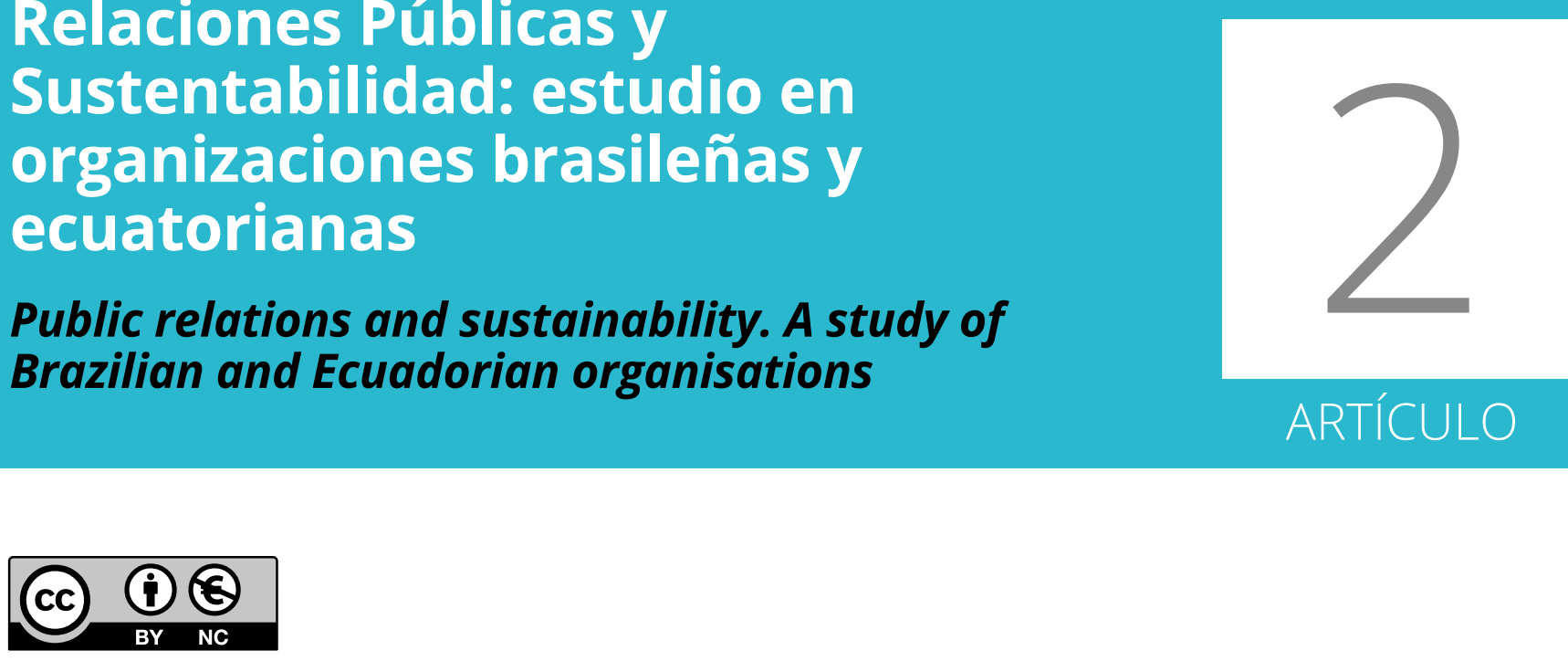

\section{María Aparecida Ferrari}

Universidade de São Paulo

Libre-docente por la Universidade de São Paulo, 2017. Doctora y Maestra en el área de Ciencias de la Comunicación de la Universidade de São Paulo, Brasil. Autora de libros sobre relaciones públicas y comunicación en Brasil, Argentina y Perú y autora de docenas de capítulos en libros publicados en los Estados Unidos, países de Europa y América Latina. Su línea de investigación abarca los temas: comunicación intercultural, interculturalidad, cultura organizacional, enseñanza y aprendizaje en relaciones públicas.

maferrar@usp.br

ORCID: 0000-0001-6873-6071

\section{Ana María Durán}

Universidad del Azuay

Profesora investigadora titular en la Escuela de Comunicación de la Universidad del Azuay. Coautora de los libros "Corporate Social Responsibility and Corporate Governance: Concepts, Perspectives and Emerging Trends in Ibero-America" y "Tejiendo sustentabilidad desde la Comunicación en Amèrica Latina. Sus lìneas de investigación están relacionadas con Relaciones Públicas, Comunicación estratègica, Comunicaciòn Organizacional, Responsabildiad Social Empresarial y Sostenibilidad.

aduran@uazuay.edu.ec

ORCID: 0000-0003-0887-2754

Fecha de recepción: 19 de julio de 2018 / 3 de septiembre de 2018 


\section{Resumen}

Se analiza comparativamente el ejercicio de las relaciones públicas y la sustentabilidad en organizaciones brasileñas y ecuatorianas. El estudio fue estructurado en tres etapas: revisión bibliográfica; investigación cuantitativa con encuestas a 177 responsables de comunicación de ambos países e investigación cualitativa con 18 entrevistas a profesionales seleccionados de la misma muestra. Los resultados indican que todavía es necesario trabajar para que la sustentabilidad se afiance en las empresas de ambos países y que el área de comunicación es esencial para aumentar el sentido de pertenencia de los empleados, reforzar la identidad corporativa y estrechar lazos con los diferentes stakeholders, apuntalando con ello el proceso de sustentabilidad.

\section{PALABRAS CLAVE}

Relaciones Públicas, comunicación, sustentabilidad, responsabilidad social, gestión de comunicación, comunicación simétrica

\section{Abstract}

We conducted a comparative analysis of public relations practices in sustainability processes in Brazilian and Ecuadorian organisations. The study was structured in three phases. The first was a bibliographic review. The second was a quantitative study with the participation of 177 organisations, 123 from Brazil and 54 from Ecuador. The third phase was qualitative and consisted of 18 interviews with heads of communication in the organisations that participated in the study. The results indicate that there is still progress to be made in both countries in terms of consolidating the sustainability process in many companies and making communication an essential field for fostering sustainability practices, increasing a sense of belonging among employees, reinforcing corporate identities and strengthening ties with company stakeholders.

\section{KEYWORDS}

Public relations, communication, sustainability, social responsibility, communication management, symmetric communication

\section{INTRODUCCIÓN}

En comparación con Norteamérica y el continente europeo, Latinoamérica es una región, cuya mayoría de países se caracteriza por economías emergentes, políticas gubernamentales, laborales y ambientales deficientes y limitadas, así como por una baja participación de la sociedad civil (Crespo Razeg, 2010). No obstante, en los últimos años la sustentabilidad ha sido determinante para las organizaciones y la sociedad latinoamericana en general, una vez que se ha convertido en un valor esencial para las relaciones con sus públicos.
Este estudio pretende llenar un vacío que existe en la literatura de relaciones públicas, comunicación y sustentabilidad en América Latina, una vez que se minimiza la importante contribución de estas disciplinas tanto para legitimar las empresas, cuanto para afianzar su relacionamiento con los públicos de interés. Para ese efecto, se entienden las relaciones públicas como una filosofía de relacionamiento y un conjunto de acciones que promueven la conformación de redes de interacciones, retroacciones, determinaciones, acontecimientos que se materializan en la cotidianidad. De estas articulaciones, negociaciones, disputas y construcciones de sentido, resultan inevitables los conflictos, el desorden, la desorganización 
y las acciones de relaciones públicas para la construcción de nuevos órdenes organizacionales (Ferrari y França 2011).

Fue a partir de 1990 que la región evidenció, principalmente en Brasil, Argentina y Chile, un importante impulso en la puesta en marcha de proyectos de responsabilidad social y sustentabilidad (Lozano, 2000). Según Torresano, (2012) el Ecuador fue una excepción a este impulso debido a su realidad económica y política, una vez que en la mencionada década las empresas ecuatorianas estaban enfocadas en superar el déficit económico y evitar la quiebra, dejando los intereses sociales como una actividad secundaria (Crusellas, 2014). Las iniciativas de las empresas en América Latina empiezan a multiplicarse, mostrando una tendencia de crecimiento exponencial a partir del año 2006 al 2010 (Sierra, Benau, \& Zorio, 2014) y entre los años 2009 y 2015 surgen las publicaciones regionales relacionadas al Global Compact (Centro Regional de Apoyo para América Latina y el Caribe, 2016).

Vale mencionar que fue en Brasil que se difundió en 1960 el primer concepto de responsabilidad social, por medio de la creación de asociaciones y entidades que se dedicaron a capacitar a ejecutivos y empresas para procesos de responsabilidad social. No obstante fue en la década de los años 1990 que entidades como ETHOS, CEBEDS y AKATU fueron creadas con el objetivo de brindar asesoría a los ejecutivos, influenciar en las políticas públicas y ofrecer herramientas para la implementación de nuevos modelos de gestión. CEBEDS fue la primera institución en Brasil que trató de la sustentabilidad utilizando el concepto Triple Bottom Line, que guía la actuación de las empresas sobre la base de tres pilares: económico, social y ambiental.
Hay que reconocer que en el Ecuador poco se ha difundido la filosofía de la sustentabilidad, incluso fue tardía la llegada del concepto de responsabilidad social. En la década de 1980 se comienza a discutir sobre ella como una iniciativa privada, cuyo mejor foro fueron las cámaras de la producción, a las que se sumaron organismos locales de desarrollo regional y gobiernos seccionales para consolidar las primeras entidades preocupadas por el tema. En 2011 se creó en el país la Red Pacto Global Ecuador, cuyo fin es promover los diez principios del Pacto Global de las Naciones Unidas en el país. En 2018 la red está conformada por 125 miembros entre empresas del sector público, privado, organizaciones de la sociedad civil, ONG, gremios y academia, comprometidas con la aplicación de los diez principios y el respeto a los derechos humanos, estándares laborales, ambiente y la lucha contra la corrupción (Pacto Global, 2018). Sin embargo, el panorama ecuatoriano en responsabilidad social y sustentabilidad según varios estudiosos se encuentra en una etapa inicial (Lansdale \& Ramos, 2013), pese a que más de la mitad de empresas ecuatorianas realizan prácticas responsables. Según los autores, el número de empresas calificadas es reducido, no todas cumplen a cabalidad con las leyes y normas establecidas, y menos aún se puede decir que van más allá de las exigencias, lo cual es característica fundamental de las empresas socialmente responsables.

A pesar de la existencia de entidades que representan y apoyan las prácticas de sustentabilidad en las empresas en ambos los países, hay una gran escasez de estudios empíricos académicos sobre la situación y la práctica de la responsabilidad social y la sustentabilidad. Todavía no se hacen investigaciones confiables y comparables entre países, lo que hace muy difícil formular generalizaciones sobre prácticas responsables y sustentables. 
En lo que respecta a la relación entre el área de comunicación con el de responsabilidad social y el de sustentabilidad, se nota un vacío en la literatura, lo que refleja la falta de investigaciones académicas locales sobre la importancia del proceso comunicativo en las prácticas sustentables. Al no existir estudios anteriores sobre esta realidad, principalmente comparando las prácticas de organizaciones brasileñas y ecuatorianas, el estudio buscó conseguir datos para llenar esos vacíos, realizando un análisis sistemático de las investigaciones recientes, realizadas en torno a la problemática que vincula las áreas de relaciones públicas y sustentabilidad.

Una vez definida la propuesta de investigación fueron establecidos sus objetivos: identificar prácticas de relaciones públicas en los procesos de sustentabilidad en organizaciones brasileñas y ecuatorianas; conocer la interrelación entre relaciones públicas y sustentabilidad, a partir de las concepciones académicas contemporáneas; verificar si las áreas de comunicación y relaciones públicas tiene injerencia en los procesos de sustentabilidad, y comparar los resultados de la investigación obtenidos en ambos países, en cuanto a prácticas de relaciones públicas vinculadas con procesos de sustentabilidad.

\section{METODOLOGÍA}

La investigación tuvo carácter exploratorio, descriptivo y comparativo. El desarrollo del estudio fue estructurado en tres etapas: primero la recopilación de literatura especializada en relaciones públicas, comunicación, responsabilidad social y sustentabilidad y el análisis de la misma. En la segunda etapa se utilizó como instrumento cuantitativo un cuestionario on-line enviado a los comunicadores de las empresas de la muestra tomada de bases de datos de las empresas de cada país construidas por las autoras, como resultado se recibieron 177 cuestionarios completadas (123 de Brasil y 54 del Ecuador). A partir de la recepción de los cuestionarios se inició el procedimiento de extracción de información de las respectivas bases de datos, con las respuestas del Ecuador y Brasil, que fueron posteriormente ordenadas y clasificadas según el plan de codificación previamente desarrollado.

Para los análisis estadísticos se utilizaron las herramientas SPSS y Minitab. El uso de esas herramientas brindó importantes alternativas para el análisis de respuesta múltiple, el análisis de escalas multidimensionales y de distintas vertientes del análisis de varianza, lo que permitió cumplir con los objetivos planteados.

En la tercera etapa se realizaron 18 entrevistas con comunicadores que habían contestado el cuestionario (selección al azar), con el objetivo de examinar algunos datos estadísticos obtenidos en la segunda etapa. Después de la realización de las entrevistas, éstas fueran transcritas y analizadas para plantear conclusiones y recomendaciones a partir de la realidad conocida.

Del total de las 177 organizaciones respondientes, 123 están instaladas en Brasil y de éstas, 87\% (107) pertenecen al sector privado, 6\% (8) al sector público, 3\% (4) al sector mixto y 3\% (4) a ONG. De las 54 organizaciones instaladas en el Ecuador, 74\% (40) forman parte del sector privado, 24\% (13) del sector público y $1 \%$ (1) se identifica como una ONG.

En ambos países la mayoría de las organizaciones participantes se encuentran clasificadas en el sector industrial, con 55\% (68) en Brasil y $44 \%$ (24) en el Ecuador. Al sector de servicios pertenecen un 35\% (43) empresas en Brasil y $42 \%$ (23), en el Ecuador. Por último, están las empresas del sector comercial, que contó con apenas 10 \% (12) en Brasil y 14 \% (7) en 
el Ecuador. Como se puede observar, la representatividad de cada sector en los dos países es bastante similar, lo que facilita el análisis y la comparación de las prácticas de comunicación en los dos países.

\section{RESULTADOS Y DISCUSIÓN}

En los últimos años se ha aceptado como premisa que la comunicación y la sustentabilidad deben darse de forma conjunta, metafóricamente como las dos caras de una misma moneda, una vez que una depende de la otra. La gestión sustentable está asociada a la manera cómo las organizaciones se relacionan con la sociedad, con el ambiente $y$, principalmente con sus públicos. Por lo tanto, exige que los procesos de comunicación de las empresas sean transparentes, integradores entre éstas y los públicos estratégicos que orbitan a su alrededor.

Un objetivo fundamental del presente estudio fue identificar si las áreas de comunicación y sustentabilidad actuaban de forma conjunta. Los resultados revelaron que el 37\% (65) de las organizaciones en ambos países tienen las áreas de comunicación y sustentabilidad en departamentos separados, mientras que en el 31\% (55) de las organizaciones las áreas de comunicación y sustentabilidad estaban bajo un mismo departamento, como puede ser observado en la Tabla 1.

Se demuestra que es una tendencia contemporánea que las empresas perciban la necesidad de que las áreas de comunicación y sustentabilidad estén juntas, en un mismo departamento. Las motivaciones para mantener las áreas unidas pueden ser explicadas por la responsabilidad de la comunicación en difundir acciones de sustentabilidad, para que los públicos tengan informaciones uniformes, permanentes y esclarecedoras y, como resultado se obtenga opinión positiva sobre el comportamiento organizacional. Una de las entrevistadas ecuatorianas mencionó que: "el departamento de Comunicación es básicamente un proveedor de servicios y es el que sabe en qué oportunidad comunicar, qué elementos del programa deben ser informados y cómo hacerlos atractivos a los medios de comunicación".

Tres de las entrevistadas de Brasil afirmaron que al principio sus actividades estaban limitadas a los procesos comunicativos pero, con el pasar del tiempo, las organizaciones pasaron a incorporar otras áreas al departamento de comunicación, como fue la sustentabilidad. Hoy, los comunicadores se ocupan de difundir todos los proyectos de sustentabilidad y responsabilidad social de las empresas, además de cumplir también con las actividades específicas de comunicación.

Tabla 1. Relacionamiento de las áreas de comunicación y sustentabilidad

\begin{tabular}{|c|c|c|c|}
\hline \multicolumn{2}{|c|}{ Brasil } & Total \\
\hline Están bajo un mismo departamento & $41(33 \%)$ & $14(26 \%)$ & $55(31 \%)$ \\
\hline Son áreas separadas & $50(41 \%)$ & $15(28 \%)$ & $65(37 \%)$ \\
\hline No existe un departamento de sustentabilidad & $32(26 \%)$ & $25(46 \%)$ & $57(32 \%)$ \\
\hline Total & $123(70 \%)$ & $54(30 \%)$ & $177(100 \%)$ \\
\hline
\end{tabular}


Los resultados demuestran que un tercio del total de las empresas, aproximadamente 33\% en Brasil y $26 \%$ en el Ecuador han optado por unificar la comunicación y la sustentabilidad en una sola área, tomando en cuenta las convergencias y las complementariedades que existen entre ambas. Convergencias tales como la opinión de los stakeholders, su enfoque administrativo y su acción dirigida a beneficios sociales. Complementariedad porque la sustentabilidad es un paradigma de gestión que pretende entablar un equilibrio en la relación entre la organización, la sociedad, la economía y el ambiente y para ello se necesita de la comunicación para que se produzca un diálogo con sus públicos (Durán \& Mosquera, 2016).

Tomando en cuenta que el concepto de sustentabilidad aún es nuevo, en particular en el Ecuador, se consultó a los comunicadores acerca de la definición del término adoptado por sus organizaciones. En el Ecuador, una de las entrevistadas señaló que: "todo proyecto de responsabilidad social que se desarrolla debe tener sustentabilidad, entendida como permanencia en el tiempo y sustentabilidad, que significa recursos suficientes para sostenerse por sí mismo". Por su parte, otra entrevistada ecuatoriana expresó que para su organización: "la responsabilidad social es el camino que lleva a la sustentabilidad o sostenibilidad, es decir, es la herramienta que permite lograr el objetivo de mejorar los impactos económicos, sociales y ambientales de la empresa".

En el cuestionario on-line, al presentar opciones para que el comunicador señale cuál era el concepto de sustentabilidad que mejor caracterizaba su organización, los resultados fueron los siguientes: en el caso de Brasil, 44\% (54) de los comunicadores eligieron la definición "sustentabilidad es una estrategia de negocios que considera los ejes social, económico y medioambiental" y que según Elkington (2001) busca atender los tres aspectos más importantes de una organización, como demuestra la tabla 9. Los resultados numéricos de los comunicadores brasileños quizás puedan ser interpretados en relación a la situación económica, política y social que desde 2014 ha asolado el país; con la crisis económica, las empresas pasaron a priorizar la sobrevivencia del negocio y no su papel social junto a la sociedad.

En el Ecuador, la definición que recibió el primer puesto, con un 48\% (26) de los comunicadores fue "la sustentabilidad es una cultura transformadora y colaborativa cuya finalidad es movilizar la empresa para el desarrollo de productos y servicios sostenibles, contribuyendo para la protección del medioambiente, la mejora continua de la calidad de vida de las personas y la garantía de los derechos de las futuras generaciones".

La respuesta de las empresas ecuatorianas es más positiva que de las brasileñas, porque demuestra que su concepto de sustentabilidad está ajustado a una perspectiva simétrica, inclusiva y transparente, apuntando que la sustentabilidad no se refiere sólo a los objetivos de negocio, sino mira el bienestar de los empleados y de la sociedad. Puede ser que las 26 empresas ecuatorianas que han elegido la referida afirmación sean grandes en tamaño y que ya tienen implementados planes integrados de sustentabilidad en sus procesos. La Tabla 2 presenta las valoraciones.

Apenas el 13\% (7) de los comunicadores ecuatorianos consideraron la sustentabilidad como una "herramienta de posicionamiento de MKT y servicios" y sólo $2 \%$ de los comunicadores brasileños comparten esa concepción. Los datos demuestran que las organizaciones que participaron de esta investigación ya avanzaron en la concepción de la relevancia que la sus- 
Tabla 2. Concepto de sustentabilidad adoptado por la organización

\begin{tabular}{|c|c|c|c|}
\hline Concepto & Brasil & Ecuador & Total \\
\hline $\begin{array}{l}\text { Una estrategia de negocios que considera los ejes social, económi- } \\
\qquad \text { co y ambiental. }\end{array}$ & $54(44 \%)$ & $12(22 \%)$ & $66(37 \%)$ \\
\hline $\begin{array}{c}\text { Una cultura transformadora y colaborativa cuya finalidad es movi- } \\
\text { lizar la empresa para el desarrollo de productos y servicios soste- } \\
\text { nibles, contribuyendo para la protección del ambiente, la mejora } \\
\text { continua de la calidad de vida de las personas y la garantía de los } \\
\text { derechos de las futuras generaciones. }\end{array}$ & $47(38 \%)$ & $26(48 \%)$ & $73(41 \%)$ \\
\hline Es un posicionamiento de mercadeo de sus productos y servicios. & $2(2 \%)$ & $7(13 \%)$ & $9(5 \%)$ \\
\hline $\begin{array}{l}\text { Es un conjunto de normas, regulaciones y certificaciones relaciona- } \\
\text { das al ambiente, relaciones de trabajo y pago de impuestos. }\end{array}$ & $11(9 \%)$ & $4(7 \%)$ & $15(9 \%)$ \\
\hline $\begin{array}{l}\text { Es la práctica y diseminación de iniciativas para reducir costos por } \\
\text { medio de mejorías en la eficiencia y en la utilización de recursos } \\
\text { (agua y energía) y destinación adecuada de los residuos. }\end{array}$ & $9(7 \%)$ & $5(9 \%)$ & $14(8 \%)$ \\
\hline Total & $123(70 \%)$ & $54(30 \%)$ & $177(100 \%)$ \\
\hline
\end{tabular}

tentabilidad tiene para el negocio. Queda claro que aumento de ventas y posicionamiento de mercadeo ya no son suficientes para presentar la organización como sustentable junto a sus stakeholders.

Con respecto a los atributos de sustentabilidad que la alta dirección más valora, los comunicadores de Brasil mencionaron el cumplimiento de la legislación/fiscal (85\%) y la ética (82\%). En el caso del Ecuador, los resultados fueron muy semejantes, con $87 \%$ para la ética y $80 \%$ para el cumplimiento fiscal/normas. El énfasis al atributo 'ética' podría deberse a la corrupción presente en el contexto político y económico de ambos países en los últimos años. Las crisis moral y de valores hacen que las organizaciones se empeñen en exigir prácticas más éticas y transparentes en los negocios y en el cumplimiento de la legislación, como mecanismo tangible del comportamiento legal.

Para identificar los objetivos estratégicos relevantes en la gestión de la sustentabilidad se presentaron siete opciones para que los comunicadores evaluaran su grado de importancia.
El encuestado tenía que considerar una escala de siete puntos, donde el número "1" es el más importante y el número "7" es el menos importante. Así, valores próximos a "1" muestran la gran importancia dada a un determinado objetivo. Para realizar el análisis fueron calculados promedios y desviaciones estándar. Los resultados obtenidos fueron posteriormente verificados mediante la utilización del análisis de variancia para evidenciar la presencia de diferencias significativas entre Brasil y el Ecuador, tomando como el valor de corte $p<0,05$. Este aspecto puede ser verificado en la Tabla 3.

Los resultados del análisis de los datos de Brasil muestran que no hay diferencias significativas entre las organizaciones nacionales y multinacionales en todos los objetivos verificados. El análisis del Brasil para el tema de los objetivos estratégicos fue focalizado desde la perspectiva de si la organización es "brasileña" (n=76) o "extranjera" ( $n=47)$. Este análisis se realizó de ese modo, a causa de que se considera que la llegada de empresas multinacionales en Brasil aceleró la implementación del concepto de la 
Tabla 3. Objetivos estratégicos para la gestión de la sustentabilidad

\begin{tabular}{|c|c|c|}
\hline Objetivos (promedios y desviación estándar) & Ecuador & Brasil \\
\hline $\begin{array}{l}\text { Promover el desarrollo social por medio de la inclusión social y económica de } \\
\text { los menos favorecidos. } \\
\qquad(F=13,43, g \mid=1 / 171, p<0,000)\end{array}$ & $2,3(1,7)$ & $3,5(2,0)$ \\
\hline $\begin{array}{l}\text { Contribuir a la transformación de la sociedad mediante el aumento de la con- } \\
\text { ciencia de la sustentabilidad pública de la organización. }\end{array}$ & $2,7(1,9)$ & $3,0(1,8)$ \\
\hline $\begin{array}{c}\text { Difundir los valores éticos en la promoción de la sustentabilidad y ser un refe- } \\
\text { rente en el tema. }\end{array}$ & $2,3(1,9)$ & $2,4(1,7)$ \\
\hline $\begin{array}{c}\text { Asegurar que la producción y el consumo de sus productos y servicios generen } \\
\text { un menor impacto en la cadena productiva y en la sociedad. }\end{array}$ & $2,3(1,7)$ & $2,8(1,9)$ \\
\hline $\begin{array}{l}\text { Generar una ventaja competitiva para el negocio, ampliar el mercado, mejorar la } \\
\text { posición estratégica en la sustentabilidad. } \\
\qquad(\mathrm{F}=8,57, \mathrm{gl}=1 / 171, \mathrm{p}<0,004)\end{array}$ & $2,0(1,4)$ & $2,9(1,9)$ \\
\hline $\begin{array}{l}\text { Administrar los intereses de los públicos estratégicos y coordinar las demandas } \\
\qquad y \text { objetivos estratégicos del negocio. } \\
\qquad(\mathrm{F}=4,36, \mathrm{gl}=1 / 171, \mathrm{p}<0,038)\end{array}$ & $2,2(1,6)$ & $2,7(1,6)$ \\
\hline $\begin{array}{l}\text { Desarrollar nuevos productos y procesos orientados a la sustentabilidad. } \\
\qquad(F=13,45, g \mid=1 / 171, p<0,000)\end{array}$ & $2,2(1,6)$ & $3,3(1,9)$ \\
\hline
\end{tabular}

sustentabilidad, y en general influenció el panorama brasileño en temas de comunicación, responsabilidad social y sustentabilidad.

El objetivo que obtuvo el mejor puntaje según los comunicadores brasileños fue "difundir los valores éticos en la promoción de la sustentabilidad y ser un referente en el tema" con 2,4, mientras que los comunicadores en el Ecuador valoraron con 2,3.

Para los encuestados brasileños, los objetivos "Administrar los intereses de los públicos estratégicos y coordinar las demandas y objetivos estratégicos del negocio" y "Asegurar que la producción y el consumo de sus productos y servicios generen un menor impacto en la cadena productiva y en la sociedad", fueron puntuados con 2,7 y 2,8 respectivamente, lo que demuestra coherencia con la definición de sustentabilidad adoptada.
Los comunicadores del Ecuador puntuaron como más importante $(2,0)$ el objetivo "generar una ventaja competitiva para el negocio, ampliar el mercado, mejorar la posición estratégica en la sustentabilidad", demostrando que todavía las organizaciones que participaron en la investigación buscan con la gestión de la sustentabilidad mejorar su actuación en los negocios, más que adoptar un comportamiento responsable en los ámbitos social, ambiental y económico. Una entrevistada ecuatoriana destacó la importancia de la sustentabilidad para adquirir ventaja competitiva cuando afirmó que: "no solo se trata de mantener el negocio, sino de diferenciarse de la competencia por acciones sostenibles en el tiempo y que generan desarrollo para los grupos de interés con los cuales la empresa interactúa".

Siguen como objetivos mejor puntuados por los comunicadores ecuatorianos (2,2) "administrar los intereses de los públicos estratégicos y 
Tabla 4. Percepción de la alta dirección acerca de los impactos de comunicación en los procesos de la sustentabilidad

\begin{tabular}{|c|c|c|c|} 
& \multicolumn{2}{c|}{ Brasil } \\
\hline $\begin{array}{c}\text { Considera que la empresa debe trabajar en fun- } \\
\text { ción de sus accionistas y no destinar recursos a } \\
\text { asuntos relacionados con otros sectores. }\end{array}$ & $4(7,4 \%)$ & $8(6,5 \%)$ & $12(6,8 \%)$ \\
\hline $\begin{array}{c}\text { Cuando hay la disponibilidad económica, la em- } \\
\text { presa usualmente atiende solicitudes de organis- } \\
\text { mos que representan grupos vulnerables de la } \\
\text { sociedad. }\end{array}$ & $6(11,1 \%)$ & $13(10,6 \%)$ & $19(10,73 \%)$ \\
\hline $\begin{array}{c}\text { La empresa incluye en su plan estratégico accio- } \\
\text { nes y colaboraciones para aportar en causas de } \\
\text { su interés y de determinados grupos. }\end{array}$ & $5(9,3 \%)$ & $24(19,5 \%)$ & $29(16,4 \%)$ \\
\hline $\begin{array}{c}\text { En forma sistemática la empresa establece alian- } \\
\text { zas y trabaja en proyectos alineados con su misión } \\
\text { y que tienen clara incidencia en lo social, ambien- } \\
\text { tal y/o económico. }\end{array}$ & $39(72,2 \%)$ & $78(63,4 \%)$ & $117(66,1 \%)$ \\
\hline
\end{tabular}

coordinar las demandas y objetivos estratégicos del negocio" y "desarrollar nuevos productos y procesos orientados a la sustentabilidad". Los mencionados objetivos están muy relacionados con metas del negocio de las organizaciones y no se enfocan hacia el concepto de sustentabilidad que fue anotado por ellos, lo que demuestra que se puede tener una concepción ideal de la sustentabilidad y, sin embargo, señalar las metas de forma que responden a una situación en la que es el negocio el que se privilegia.

En seguida se buscó identificar la percepción de los miembros de la alta gerencia acerca de los impactos de la comunicación en la sustentabilidad, según los comunicadores de ambos países, según se observa en la Tabla 4.

El $66 \%$ de los comunicadores de ambos países (72\% el Ecuador y 63\% Brasil) señalaron que "en forma sistemática la empresa establece alianzas y trabaja en proyectos alineados con su misión y que tienen clara incidencia en lo social, ambiental y/o económico". La alternativa elegida como la más valorada por los comunicadores de Brasil y del Ecuador indica que la alta dirección comprende la importancia de trabajar con los tres ejes de triple bottom line; sin embargo en respuestas anteriores se pudo notar la mayor importancia otorgada en la práctica al eje económico, en perjuicio, por ejemplo, del eje social. La siguiente práctica más señalada fue "la empresa incluye en su plan estratégico acciones y colaboraciones para aportar en causas de su interés y de determinados grupos" por 16\% (29) de los comunicadores de ambos países.

Esta cuestión puede ser considerada la 'médula' de la presente investigación, una vez que demuestra de manera contundente que la mayoría de los comunicadores (117) de ambos países percibe que sus organizaciones tratan de alinear sus proyectos de sustentabilidad a sus metas organizacionales. Tal comportamiento organizacional tiene como objetivo ser legal y legítimo, una vez que la actuación organizacional está de acuerdo con la legislación vigente, así como también trata de establecer alianzas para el desarrollo de proyectos que estén alineados con la filosofía y la misión organizacional. 
Tabla 5. Planificación del trabajo del comunicador

\begin{tabular}{|c|c|c|c|}
\hline Planificación del trabajo & $\begin{array}{c}\text { Brasil } \\
(\mathrm{n}=123)\end{array}$ & $\begin{array}{l}\text { Ecuador } \\
(n=54)\end{array}$ & Total \\
\hline $\begin{array}{c}\text { Se procura destinar todo el tiempo posible a la optimiza- } \\
\text { ción de la productividad de la empresa y al incremento de } \\
\text { sus utilidades. }\end{array}$ & $6(5 \%)$ & $8(15 \%)$ & $14(8 \%)$ \\
\hline $\begin{array}{c}\text { Se permite que se atienda y dé respuesta a solicitudes de } \\
\text { diferentes sectores sociales sin prever beneficios para la } \\
\text { organización. }\end{array}$ & $3(2 \%)$ & $6(11 \%)$ & $9(5 \%)$ \\
\hline $\begin{array}{l}\text { Se incorporan actividades que buscan solucionar proble- } \\
\text { mas del entorno o de grupos sociales vinculados a la em- } \\
\text { presa, con los que se dialoga. }\end{array}$ & $70(57 \%)$ & $9(17 \%)$ & $79(45 \%)$ \\
\hline $\begin{array}{l}\text { Se incluye regularmente la gestión y evaluación de estrate- } \\
\text { gias enfocadas al incremento de la productividad, el mejo- } \\
\text { ramiento de la calidad de vida de sectores vinculados y/o el } \\
\text { cuidado del ambiente, con base en la interacción con otras } \\
\text { organizaciones. }\end{array}$ & $44(36 \%)$ & $31(57 \%)$ & $75(42 \%)$ \\
\hline
\end{tabular}

Flores, Ogliastri, Peinado-Varas y Petri (2007) afirman que en el caso de América Latina, las empresas en la mayoría de países de la región, están regidas por los códigos de comercio y de sus estatutos, y en los casos de empresas que emiten títulos-valores, por las leyes de mercados de capitales. En general se establecen regulaciones sobre el manejo prudente de los recursos de la empresa, pero no existe la obligación de la maximización de beneficios. Los gerentes de empresa tienen la facultad de decidir sobre la administración de los recursos y pueden, discrecionalmente, dedicar parte de ellos a actividades que beneficien a terceros teniendo en cuenta también que no perjudiquen a la empresa.

Por fin, la investigación trató de verificar cómo el comunicador planificaba su trabajo, ya que la planificación y el control son la base de una gestión efectiva de la comunicación y de ellos depende el enfoque que se persigue y por consiguiente los resultados que se logren, como pude ser visto en la Tabla 5.
En Brasil 57\% (70) de los comunicadores afirmaron que en su trabajo cotidiano "incorporan actividades que buscan solucionar problemas del entorno o de grupos sociales vinculados a la empresa, con los que se dialoga". Significa que la comunicación adquiere un rol estratégico, principalmente por su preocupación respecto a los stakeholders y a mantener el diálogo con ellos, estableciéndose una vía bidireccional, en la cual la organización y sus públicos buscan el consenso y el equilibrio de sus necesidades.

En contraste, en el Ecuador 57\% (31) de los comunicadores afirmaron que "incluyen regularmente la gestión y evaluación de estrategias enfocadas al incremento de la productividad, el mejoramiento de la calidad de vida de sectores vinculados y/o el cuidado del ambiente, con base en la interacción con otras organizaciones". El resultado de los comunicadores ecuatorianos cumple con el modelo asimétrico propuesto por Grunig y Hunt (1984) en que la organización está interesada en que los públicos ajusten sus expectaciones a las de la organización, y no al revés. Hay que considerar que, frente al escenario latinoamericano en que las 
crisis económicas son frecuentes y el sistema político vulnerable, las prácticas apuntadas por los comunicadores del Ecuador les sirven para demostrar que la comunicación es un proceso que está permanente y claramente orientado a colaborar para el mejoramiento de la calidad de vida de sectores vinculados y/o el cuidado del ambiente y menos de relacionarse con sus públicos y persuadirlos a trabajar en forma conjunta por una mejor calidad de vida que incluya la percepción de sus públicos estratégicos.

En los resultados obtenidos se observa que en varios aspectos hay semejanzas en la forma con la cual los departamentos de comunicación de ambos países desarrollan sus acciones y, en otros se observó diferencias que tienen su explicación en las políticas públicas e institucionales, como también de los factores culturales que impactan en el cotidiano de los contextos ecuatoriano y brasileño.

Las diferencias y similitudes son resultado de los cambios que las empresas enfrentan; un 'cambio sísmico', sin precedentes, ante los desafíos sociales y ambientales, de la innovación técnica y de los modelos de negocio.

Aunque ambos países pertenecen a una región bastante atribulada por crisis económicas, políticas e institucionales, es posible analizar, por los resultados, que parte relevante de las organizaciones encuestadas está trabajando para hacer la diferencia en la sociedad cuando establecen la sustentabilidad como estrategia de los negocios y reconocen en la comunicación un proceso de creación de sentido para que los stakeholders sean participantes del esfuerzo organizacional.

También está claro que los países de América Latina son caracterizados por rasgos culturales que influyen en los modelos de gestión y en la forma cómo y qué comunican las organizaciones; en las respuestas de los encuestados se observó la importancia que todavía tiene la jerarquía descendente, el personalismo, la tendencia al autoritarismo, la falta de creatividad e innovación; pero también se notó que en ambos países se encuentran organizaciones y grupos económicos innovadores que son más abiertos a reflexionar en el beneficio común y orientados hacia el futuro como forma de sobrevivir en tiempos de globalización y vulnerabilidad.

\section{CONCLUSIONES}

Los resultados de la presente investigación, al confrontar la realidad de dos países latinoamericanos con grandes diferencias en términos de tamaño de territorio geográfico, número de habitantes, rasgos culturales y trayectoria, tanto relacionada con el desarrollo de las Relaciones Públicas y comunicación cuanto con la sustentabilidad, dejan ver semejanzas, desafíos, avances y problemáticas comunes que trascienden las diferencias mencionadas.

De acuerdo con los resultados observados en las 177 organizaciones participantes de la investigación, los beneficios del correcto concepto y práctica de comunicación y sustentabilidad para el negocio de la organización son factores de apalancamiento para la competitividad, en lugar de actividades utilizadas como herramientas del marketing o la filantropía. También está claro que las acciones de comunicación y sustentabilidad varían según las características del negocio y de la industria, dependiendo tanto de elementos como valores, modelo de liderazgo, cultura interna y externa, como de estrategias de negocios.

Es un hecho que se encontraron concepciones reduccionistas, sean por parte de ejecutivos de las organizaciones, como también de parte de la sociedad, que aún miran las relaciones públi- 
cas como una instancia operativa, cuya función está relacionada con la difusión a través de los medios de comunicación y que la sustentabilidad es un conjunto de prácticas orientadas a fidelizar clientes y mejorar la imagen de las empresas. Sin embargo, los datos recopilados indican que en ambos países se está pasando por un período de transición importante, para dar paso a una mentalidad más proclive a una comunicación verdaderamente bidireccional, que beneficie tanto a las organizaciones cuanto a los públicos involucrados.

La investigación encontró que en sólo 55 (31\%) del total de 177 empresas, las áreas de comunicación y sustentabilidad están bajo un mismo departamento. En 65 (37\%) de empresas las áreas de comunicación y sustentabilidad estaban separadas y en 57 (32\%) simplemente no existía departamento de sustentabilidad. En el caso del Ecuador es bastante expresivo el número de organizaciones (46\%) que todavía no han estructurado el área o sector de sustentabilidad, lo que denota que aún hay un largo camino a seguir en términos de pensar el negocio con fines que van más allá de las ganancias y que las prácticas sustentables sean parte del propósito de la organización y no solamente un discurso que no se aplique en la cotidianidad de la empresa.

Éstos resultados están alineados al concepto de sustentabilidad adoptado por las organizaciones de ambos los países. Los dos conceptos más señalados por el total de los comunicadores son los que demuestran un avance en términos de filosofía organizacional. En Brasil 44\% (55) empresas mencionaron la sustentabilidad como una estrategia de negocios, considerando los tres ejes de Elkington (1994), mientras que 38\% (44) señalaron que la sustentabilidad es una cultura transformadora, tanto para la empresa como para la sociedad. La división entre el primero y el segundo concepto de sustentabilidad quizás esté relacionada a las empresas nacionales e internacionales, una vez que el último grupo ya viene trabajando la temática por medio de las políticas establecidas desde la matriz. En el caso del Ecuador, los puntajes fueron exactamente al contrario, dónde $48 \%$ (26) de los ecuatorianos valoraron la sustentabilidad como cultura transformadora y 22\% (12) como estrategia de negocios.

La evaluación de las investigadoras es positiva en cuanto a ese resultado, una vez que los conceptos menos mencionados fueron los que identifican la sustentabilidad como herramienta de marketing y de reducción de costos en la empresa. Eso indica que está presente una mirada ética y se puede prever que las futuras generaciones superarán las miras a buscar solamente resultados inmediatos para los negocios, trascendiendo hacia la búsqueda del bienestar social, ambiental y económico para la comunidad en su conjunto. 


\section{BIBLIOGRAFÍA}

Centro Regional de Apoyo para América Latina y el Caribe. (2016). Panorama Social de América Latina. Recuperado el 14 de febrero de 2018, de http://repositorio.cepal.org/bitstream/handle/11362/41598/4/ S1700567_es.pdf

Crespo Razeg, F. (2010). Entre el Concepto y la Práctica: Responsabilidad Social Empresarial. Estudios Gerenciales, 119-130.

Crusellas, M. (2014). La responsabilidad social empresarial (RSE) como estrategia de Relaciones Públicas, propuesta para empresas privadas de Cuenca, Ecuador. Cuenca: Universidad de Cuenca.

Durán, A., \& Mosquera, P. (2016). Relaciones Públicas y Sustentabilidad: Disciplinas convergentes y complementarias. Quito: Red Internacional de Investigación de Gestión de la Comunicación (XESCOM). Recuperado el 21 de Febrero de 2018, de https://xescom2016.files.wordpress. com/2017/01/documento-xescom.pdf

Elkington, J. (1994). Towards the sustainable corporation: Win-win-win business strategies for sustainable development. California Management Review, 36(2), 90-100.

Elkington, J. (2001). Canibais com garfo e faca. São Paulo: Makron Books.

Ferrari, M. A. Ferrari, M.A. y França, F. (2011). Relaciones Públicas: naturaleza, función y gestión de las organizaciones contemporáneas. Buenos Aires: La Crujía.

Flores, J., Ogliastri, E., Peinado-Varas, E., \& Petri, I. (2007). El argumento empresarial de la RSE: 9 casos de América Latina y el Caribe. Banco Interamericano de Desarrollo, Washington.

Lansdale, D., \& Ramos, C. (2013). Responsabilidad Social Empresarial: Una herramienta para crear sostenibilidad económica, social y ecológica en las empresas que operan en el Ecuador. Quito: Universidad San Francisco de Quito. Obtenido de http://repositorio.usfq.edu.ec/handle/23000/2315

Lozano, J. M. (2000). La empresa en la sociedad: responsabilidades éticas. América Latina y España: un futuro compartido. 129-154.

Pacto Global. (15 de febrero de 2018). Pacto Global de las Naciones Unidas Ecuador. Obtenido de http://www.pactoglobal-ecuador.org

Sierra, Benau, \& Zorio. (2014). Credibilidad en Latinoamérica del informe de responsabilidad social corporativa. RAE - Revista de Administração de Empresas, 28-38.

Torresano, M. (2012). Estudio de Responsabilidad Social en Empresas del Ecuador. Quito: NOCIÓN IMPRENTA. 